\title{
Brasil: tabagismo e consumo de bebida alcoólica nos últimos dez anos (vigitel) e o papel do Cirurgião-Dentista na prevenção do câncer bucal
}

\author{
Brazil: smoking and alcoholic beverage consumption in the last ten years (vigitel) and the role of \\ the Dental Surgeon in the prevention of oral cancer
}

Brasil: fumar y consumo de bebidas alcohólicas en los últimos diez años (vigitel) y el papel del Cirujano Dental en la prevención del cáncer oral

\author{
Ana Carolina Amorim de Sousa Lopes \\ ORCID: https://orcid.org/0000-0001-9060-2782 \\ Universidade Potiguar, Brasil \\ E-mail: anacarolinaas_lopes@hotmail.com \\ Kaio César de Oliveira Barbosa \\ ORCID: https://orcid.org/0000-0001-5404-180X \\ Universidade Potiguar, Brasil \\ E-mail: kaiortis@hotmail.com \\ Maria Luísa Faria Barroso \\ ORCID: https://orcid.org/0000-0002-2818-6594 \\ Universidade Potiguar, Brasil \\ E-mail: luisafariaa@outlook.com \\ Ana Larissa Fernandes de Holanda Soares \\ ORCID: https://orcid.org/0000-0002-3740-7862 \\ Universidade Federal do Rio Grande do Norte, Brasil \\ E-mail: pedrolauramafaldo@gmail.com
}

\begin{abstract}
Resumo
O presente trabalho objetivou discutir a frequência estimada do tabagismo e consumo de bebida alcoólica nos últimos 30 dias, por sexo, faixa etária e grau de escolaridade no Brasil nos últimos dez anos, e analisar o papel do cirurgiãodentista da atenção básica frente aos principais fatores de risco para o câncer bucal. Foi realizado por meio de um estudo ecológico com dados do VIGITEL entre os anos de 2009 e 2019, e que teve como resultado uma diminuição positiva no número de tabagistas, decrescendo de 15,5 para 9,8\%, e no número de consumidores de álcool, principalmente entre os adultos jovens, houve um aumento de 22,9 para 26,3\%. Na análise dos profissionais na atenção primária à saúde ficou clara a necessidade de políticas públicas que deem sustentação as ações de prevenção do câncer bucal e a atualização do cirurgião-dentista diante do combate a essa patologia. Apesar da redução no percentual de fumantes no Brasil nos últimos anos, o consumo de bebida alcoólica ainda permanece sem responder às iniciativas governamentais direcionadas ao seu combate. O cirurgião-dentista representa um importante ator, dentro da equipe multiprofissional, na sensibilização da população para aquisição de hábitos saldáveis e prevenção do câncer de boca.
\end{abstract}

Palavras-chave: Câncer bucal; Odontólogos; Fumar.

\begin{abstract}
The present study aimed to discuss the estimated frequency of smoking and alcohol consumption in the last 30 days, by sex, age group and education level in Brazil in the last ten years, and to analyze the role of the dentist in primary care in relation to the main risk factors for oral cancer. It was carried out through an ecological study with data from VIGITEL between the years 2009 and 2019, which resulted in a positive decrease in the number of smokers, decreasing from 15.5 to $9.8 \%$, and in the number of alcohol consumers especially among young adults, there was an increase from 22.9 to $26.3 \%$. In the analysis of professionals in primary health care, the need for public policies to support oral cancer prevention actions and the updating of dentists in the fight against this pathology was clear. Despite the reduction in the percentage of smokers in Brazil in recent years, the consumption of alcoholic beverages remains unresponsive to government initiatives aimed at combating it. The dental surgeon represents an important actor, within the multiprofessional team, in sensitizing the population to acquire healthy habits and prevent oral cancer.
\end{abstract}

Keywords: Oral câncer; Odontologists; Smoking. 


\begin{abstract}
Resumen
Este estudio tuvo como objetivo discutir la frecuencia estimada de consumo de tabaco y alcohol en los últimos 30 días, por sexo, grupo de edad y nivel de educación en Brasil en los últimos diez años, y analizar el papel de los odontólogos de atención primaria en relación con los principales factores de riesgo de cáncer oral. Se realizó a través de un estudio ecológico con datos de VIGITEL entre 2009 y 2019, que resultó en una disminución positiva en el número de fumadores, disminuyendo del 15,5 al 9,8\%, y en el número de consumidores de alcohol, especialmente entre los adultos jóvenes, hubo un aumento del 22,9 al 26,3\%. En el análisis de los profesionales de la atención primaria de salud, quedó patente la necesidad de políticas públicas que apoyen las acciones de prevención del cáncer bucal y la actualización de los odontólogos en la lucha contra esta patología. A pesar de la reducción del porcentaje de fumadores en Brasil en los últimos años, el consumo de bebidas alcohólicas sigue sin responder a las iniciativas gubernamentales destinadas a combatirlo. Los cirujanos dentistas representan un actor importante dentro del equipo multiprofesional en la sensibilización de la población sobre la adquisición de hábitos saludables y la prevención del cáncer bucal.
\end{abstract}

Palabra clave: Cáncer oral; Odontólogos; Fumar.

\title{
1. Introdução
}

É denominado câncer, doenças que possuem uma proliferação desordenada de células invasoras de órgãos e tecidos, e uma grande capacidade de se espalhar de forma rápida pelo corpo (Brasil, 2019).

Em 2018, o oitavo tipo de neoplasia que mais matou homens no Brasil, chegando a quase cinco mil o número de mortos foi o câncer bucal. Este, consiste em um tumor maligno que pode afetar lábio, bochecha, língua, assoalho, palato e gengiva. Na região sudeste esse tumor chega a ser o quarto mais comumente encontrado em pessoas do sexo masculino acima de quarenta anos, e geralmente leucodermas. Já em mulheres há uma diminuição na posição desse ranking, aparecendo como décimo terceiro colocado, logo, estando fora da listagem dos dez mais letais. A nível mundial, neste mesmo ano, houve o surgimento registrado de 354 mil novos casos, com prevalência maior no Sul da Ásia (INCA, 2020).

Segundo o Instituto Nacional do Câncer, o carcinoma epidermóide, que é o tumor mais frequente (cerca de 95\% dos casos) no câncer bucal, pode ser classificado em: pouco, moderadamente e bem diferenciado. Apesar desse possuir maior incidência, pode-se encontrar também outros tipos de tumores na cavidade oral, tais como: sarcomas, tumores ligados às glândulas salivares e o melanoma de mucosa. Embora essa doença possa se manifestar em toda a boca, a língua, o assoalho bucal e o lábio inferior, são em ordem decrescente as áreas mais acometidas por ela (Andrade; Oliveira; Santos, 2015).

Partindo para a análise das características clínicas, é normalmente notada a presença de lesões leucoeritoplásicas e ulceradas. Já no que diz respeito à profundidade, crescimento, implantação, consistência e forma, há uma grande variabilidade (Cedraz et al, 2016).

Pesquisas mostram que atualmente já se tem o conhecimento que diversos são os fatores etiológicos do câncer bucal, sendo eles: raios solares, dieta desequilibrada, imunidade deficiente, alcoolismo e tabagismo. Porém, é preciso ressaltar que, esses dois últimos fatores citados são exponencialmente relevantes e contribuintes no surgimento dessa patologia (Martins et al, 2015). Baseando-se nessa informação, a Sociedade Brasileira de Cirurgia de Cabeça e Pescoço (SBCPP), realizou um estudo epidemiológico comprovando que de fato, a exposição ao tabaco e ao álcool são os principais fatores causais associados ao desenvolvimento do carcinoma espinocelular, que é o tumor mais frequente do trato aerodigestivo superior (Souza et al, 2016).

Sabe-se que o tabaco é um produto agrícola processado a partir das folhas de plantas do gênero Nicotiana L., e que sua utilização pode ser feita por meio de várias formas, como: cigarro, cachimbo, charuto, rapé e fumo mascado. Atualmente, são mais de 60 substâncias cancerígenas encontradas no fumo (Scully; Kirby, 2014).

Ainda sobre a nicotina, sua participação na carcinogênese se deve por atuar como intermediária de macromoléculas que produzem nitrosaminas (compostos químicos cancerígenos específicos do tabaco). Além de, na sua metabolização no fígado, formar um subproduto, que participa da síntese de substâncias cancerígenas. Recentemente, estudos evidenciaram que a 
nicotina possui propriedades de angiogênese (formação de novos vasos) que favorece a proliferação das células cancerosas (American Cancer Society, 2020)

O álcool etílico ou etanol é uma substância orgânica obtida da fermentação microbiana de açúcar (amido, grãos e carboidratos nas frutas), ele é encontrado na cerveja (cerca de 10\%), vinho (16\%) e vodka (40\%). O teor alcoólico de tais bebidas associado ao alto nível de ingestão propicia a relação do câncer bucal e etilismo (Ogden, 2018). Em geral, o fator mais importante no aumento do risco de câncer é a quantidade de álcool consumida ao longo do tempo, e não o tipo de bebida alcoólica.

Partindo para a análise química, é de conhecimento que o acetaldeído é resultado metabólico primário do etanol na sua rota de conversão de ácido acético. É um produto tóxico e carcinogênico humano que pode danificar o DNA e as proteínas. Além disso, o álcool dificulta a capacidade do organismo em quebrar e absorver vários nutrientes: vitamina A, vitaminas do complexo B, vitamina C, vitamina D, vitamina E e carotenóides. Que atuam como protetores contra o câncer bucal, realizando a oxidação, que é um processo que gera substâncias reativas do oxigênio que podem danificar o DNA, as proteínas e os lipídios do corpo (OGDEN, 2018).

Nesta perspectiva, a associação dos hábitos de beber e fumar multiplica em até 20 vezes a chance de uma pessoa saudável desenvolver câncer bucal, ainda segundo a Sociedade Brasileira de Cirurgia de Cabeça e Pescoço (SBCCP). Além disso, pesquisas anteriores indicaram que a falta de conscientização pública sobre os sinais e fatores de risco dessa doença, podem contribuir para o diagnóstico tardio e, consequentemente, pior prognóstico (Hassona et al, 2015).

Neste sentido, a prevenção e o diagnóstico precoce são de extrema importância para uma diminuição nas taxas de casos. Visando esses resultados e sabendo que essa patologia é crônica e se configura como caso de saúde pública, o SUS tem implantado nos últimos anos políticas voltadas à promoção de saúde e educação, com enfoque principalmente para o consumidor de álcool e tabaco, já que esses são os maiores responsáveis pelo surgimento da doença (Martins et al, 2015).

Com o olhar voltado para essas ações de prevenção, o Ministério da Saúde lançou em 2006, após a aprovação da CONEP (Comissões do Conselho Nacional de Saúde), o VIGITEL, que consiste em uma espécie de pesquisa que compõe o Sistema de Vigilância de Fatores de Risco, e que é realizada pela SVS (Secretaria de Vigilância em Saúde). Essa pesquisa atua nas capitais do país e no Distrito Federal, via linha telefônica (são escolhidas para o telefonema pessoas aleatórias que possuam 18 anos ou mais e que tenham linha fixa de telefone) e tem como objetivo, após um questionário rápido com perguntas mais voltadas a saúde, monitorar a frequência e a distribuição de fatores de risco e proteção para doenças crônicas não transmissíveis. Entre as doenças monitoradas estão: as cardiovasculares, diabetes, obesidade e os diversos tipos de câncer. Essa coleta de dados divulgada ao final de cada ano, é importante pois serve como base para a elaboração, por parte do governo, de atividades que inibam a ocorrência dessas doenças, evitando assim o agravamento de saúde da população (Brasil, 2020).

O presente estudo teve como objetivos discutir a frequência estimada de tabagismo e o consumo de bebidas alcoólicas nos últimos 30 dias no Brasil, por sexo, faixa etária e grau de escolaridade, entre os anos de $2009-2019$ e analisar o papel do cirurgião-dentista, inserido em uma equipe da Atenção Primária à Saúde, na prevenção da instalação dos principais fatores de risco para o câncer bucal (tabagismo e consumo de bebidas alcoólicas).

\section{Metodologia}

O trabalho trata-se de um estudo ecológico descritivo quantitativo e revisão narrativa da literatura. Para a realização da pesquisa, os dados foram coletados a partir dos resultados do VIGITEL (Vigilância de Doenças Crônicas por Inquérito Telefônico), pesquisa feita anualmente com base em uma amostragem probabilística e sob a responsabilidade do Ministério da Saúde, selecionando uma amostra da população adulta de cada uma das capitais dos 26 estados brasileiros e do Distrito Federal. O VIGITEL tem como objetivo monitorar a frequência e a distribuição de fatores de risco e proteção para Doenças 
Crônicas Não Transmissíveis (DCNT), por meio de entrevistas telefônicas realizadas nos residentes em domicílios servidos por linhas fixas de telefone em cada cidade. Foram incluídos e analisados os dados publicados nos últimos 10 anos (período compreendido entre 2009 e 2019) referentes às variáveis dependentes e independentes descritas a seguir: Variáveis dependentes: tabagismo e consumo de bebidas alcoólicas nos últimos 30 dias. Variáveis independentes: sexo, faixa etária e nível de escolaridade.

Para discutir o papel do cirurgião-dentista, inserido em uma equipe da Atenção Primária à Saúde do Sistema Único de Saúde, no combate ao câncer de boca, a partir da prevenção do tabagismo e do consumo de bebidas alcoólicas, foi desenvolvida uma revisão narrativa da literatura, a qual foi elaborada de acordo com a busca de artigos sobre o tema nas bases de dados eletrônicas BIREME, SCIELO e LILACS e complementada em documentos e sites governamentais. Devido ao uso de dados secundários provenientes de pesquisas pré-existentes, não houve necessidade de submissão do trabalho ao Comitê de Ética em Pesquisa (CEP) da Universidade Potiguar (UnP). Em relação aos riscos e benefícios, não há riscos na pesquisa em virtude de ser um estudo baseado em dados secundários. Em relação aos benefícios, o estudo possibilitará conhecer a distribuição do tabagismo e do consumo de bebidas alcoólicas no país, os quais estão implicados com a causalidade do câncer de boca, favorecendo o planejamento futuro de ações voltadas à prevenção dessa patologia, assim como de promoção de hábitos saudáveis na população. OS dados foram analisados a partir de estatística descritiva, por meio de tabelas, tabulados em Excel 2016.

\section{Resultados e Discussão}

Tabela 1 - Percentual de fumantes no conjunto da população adulta ( $\geq 18$ anos) das capitais dos estados brasileiros e do Distrito Federal, por sexo, segundo idade e anos de escolaridade. VIGITEL, 2009-2019.

\begin{tabular}{l|l|l|l|l|l|l|l|l|l|l|l} 
VARIÁVEL & $\mathbf{2 0 0 9}$ & $\mathbf{2 0 1 0}$ & $\mathbf{2 0 1 1}$ & $\mathbf{2 0 1 2}$ & $\mathbf{2 0 1 3}$ & $\mathbf{2 0 1 4}$ & $\mathbf{2 0 1 5}$ & $\mathbf{2 0 1 6}$ & $\mathbf{2 0 1 7}$ & $\mathbf{2 0 1 8}$ & $\mathbf{2 0 1 9}$ \\
\hline FUMANTES & $\mathbf{\%}$ & $\mathbf{\%}$ & $\mathbf{\%}$ & $\mathbf{\%}$ & $\mathbf{\%}$ & $\mathbf{\%}$ & $\mathbf{\%}$ & $\mathbf{\%}$ & $\mathbf{\%}$ & $\mathbf{\%}$ & $\mathbf{\%}$ \\
\hline SIM & 15,5 & 15,1 & 21,7 & 12,1 & 11,3 & 10,8 & 10,4 & 10,2 & 10,1 & 9,3 & 9,8 \\
\hline NÃO & 84,5 & 84,9 & 78,3 & 87,9 & 88,7 & 89,2 & 89,6 & 89,9 & 89,9 & 90,7 & 90,2 \\
\hline SEXO & & & & & & & & & & & \\
\hline MASCULINO & 19,0 & 17,9 & 25,0 & 15,5 & 14,4 & 12,8 & 12,8 & 12,7 & 13,2 & 12,1 & 12,3 \\
\hline FEMININO & 12,5 & 12,7 & 18,8 & 9,2 & 8,6 & 9,0 & 8,3 & 8,0 & 7,5 & 6,9 & 7,7 \\
\hline IDADE- ANOS & & & & & & & & & & & \\
\hline 18 A 24 & 15 & 12,5 & 11,3 & 8,5 & 7,1 & 7,8 & 7,2 & 7,4 & 8,5 & 6,7 & 7,9 \\
\hline 25 A 34 & 16,4 & 17,3 & 15,4 & 11,7 & 12,1 & 11,9 & 10,5 & 9,7 & 9,6 & 9,4 & 9,2 \\
\hline 35 A 44 & 15,1 & 15,2 & 20,0 & 12,9 & 11,2 & 9,9 & 10,4 & 10,0 & 11,7 & 9,1 & 9,7 \\
\hline 45 A 54 & 19,0 & 18,3 & 33,1 & 16,0 & 15,1 & 13,2 & 12,7 & 12,6 & 11,2 & 11,1 & 10,9 \\
\hline 55 A 64 & 16,9 & 16,5 & 37,3 & 15,0 & 13,6 & 12,5 & 12,8 & 13,5 & 11,6 & 12,3 & 12,6 \\
\hline 65 E MAIS & 8,1 & 8,1 & 35,2 & 7,6 & 6,9 & 8,1 & 8,2 & 7,7 & 7,3 & 6,1 & 7,8 \\
\hline ESCOLARIDADE-ANOS & & & & & & & & & & & \\
\hline 0 A 8 & 19,3 & 18,6 & 26,1 & 16,4 & 15 & 14,1 & 14,4 & 14,3 & 13,2 & 13,0 & 13,8 \\
\hline 9 - 11 & 11,3 & 11,6 & 15,7 & 10,0 & 10,3 & 10,3 & 9,0 & 9,4 & 9,9 & 8,8 & 9,5 \\
\hline 12 E MAIS & 11,1 & 10,2 & 18 & 9,1 & 7,4 & 6,8 & 7,2 & 6,9 & 7,4 & 6,2 & 6,7 \\
\hline
\end{tabular}

Fonte: Ministério da Saúde. 
A partir dos dados do VIGITEL, foi possível perceber na tabela 1 que, a prevalência de fumantes esteve maior entre os indivíduos do sexo masculino, na faixa etária entre 45 e 54 anos e com menor número de anos de estudo. A tabela 1 demonstra também uma diminuição do percentual de fumantes na população adulta no Brasil, nos últimos 10 anos, passando de $15,5 \%$ em 2009 para 9,8\% em 2019.

Tabela 2 - Percentual de indivíduos que, nos últimos 30 dias, consumiram quatro ou mais doses (mulher) ou cinco ou mais doses (homem) de bebida alcoólica em uma mesma ocasião no conjunto da população adulta ( $\geq 18$ anos) das capitais dos estados brasileiros e do Distrito Federal, por sexo, segundo idade e anos de escolaridade. VIGITEL, 2009 - 2019.

\begin{tabular}{l|l|l|l|l|l|l|l|l|l|l|l} 
VARIÁVEL & $\mathbf{2 0 0 9}$ & $\mathbf{2 0 1 0}$ & $\mathbf{2 0 1 1}$ & $\mathbf{2 0 1 2}$ & $\mathbf{2 0 1 3}$ & $\mathbf{2 0 1 4}$ & $\mathbf{2 0 1 5}$ & $\mathbf{2 0 1 6}$ & $\mathbf{2 0 1 7}$ & $\mathbf{2 0 1 8}$ & $\mathbf{2 0 1 9}$ \\
\hline $\begin{array}{l}\text { BEBIDA } \\
\text { ALCOÓLICA }\end{array}$ & $\mathbf{\%}$ & $\mathbf{\%}$ & $\mathbf{\%}$ & $\mathbf{\%}$ & $\mathbf{\%}$ & $\mathbf{\%}$ & $\mathbf{\%}$ & $\mathbf{\%}$ & $\mathbf{\%}$ & $\mathbf{\%}$ & $\mathbf{\%}$ \\
\hline SIM & 18,9 & 18,0 & 17,0 & 18,4 & 16,4 & 16,5 & 17,2 & 19,1 & 19,1 & 17,9 & 18,8 \\
\hline NÃO & 81,1 & 82,0 & 83,0 & 81,6 & 83,6 & 83,5 & 82,8 & 80,9 & 80,9 & 82,1 & 81,2 \\
\hline SEXO & & & & & & & & & & & \\
\hline MASCULINO & 28,8 & 26,8 & 26,2 & 27,9 & 24,2 & 24,8 & 25,3 & 27,3 & 27,1 & 26,0 & 25,3 \\
\hline FEMININO & 10,4 & 10,6 & 9,1 & 10,3 & 9,7 & 9,4 & 10,2 & 12,1 & 12,2 & 11,0 & 13,3 \\
\hline IDADE - ANOS & & & & & & & & & & & \\
\hline 18 A 24 & 23 & 20,3 & 20,5 & 21,8 & 19,0 & 18,2 & 20,2 & 22,1 & 23,8 & 23 & 25,8 \\
\hline 25 A 34 & 22,9 & 23,2 & 20,7 & 24,7 & 22,7 & 23,2 & 23,5 & 25,8 & 27,7 & 24,2 & 26,3 \\
\hline 35 A 44 & 20,6 & 19,5 & 19,1 & 20,0 & 17,5 & 18 & 19,4 & 21,2 & 22,2 & 21,7 & 20,9 \\
\hline 45 A 54 & 17,0 & 16,1 & 14,8 & 16,6 & 15,0 & 15,1 & 15,5 & 18,2 & 15,8 & 14,7 & 15,8 \\
\hline 55 A 64 & 10,5 & 10,4 & 10,6 & 11,9 & 10,5 & 11,0 & 11,0 & 12,6 & 10,3 & 11,0 & 11,2 \\
\hline 65 E MAIS & 4,2 & 4,5 & 4,3 & 5,0 & 4,0 & 3,8 & 3,7 & 4,6 & 3,0 & 4,1 & 4,1 \\
\hline ESCOLARIDADE & & & & & & & & & & & \\
ANOS & & & & & & & & & & & \\
\hline 0 A 8 & 17,1 & 16 & 15,9 & 15 & 12,8 & 12,3 & 13,2 & 14,2 & 13,8 & 13,0 & 12,4 \\
\hline 9 - 11 & 19,8 & 19,2 & 17,2 & 19,4 & 17,5 & 18,4 & 18,2 & 19,2 & 20,2 & 19,1 & 20,0 \\
\hline 12 E MAIS & 23,1 & 22,3 & 20,1 & 22,0 & 19,7 & 19,5 & 20,9 & 24,0 & 22,8 & 21,2 & 23,1 \\
& & & & & & & & & & \\
\end{tabular}

Fonte: Ministério da Saúde.

De acordo com a tabela 2, o maior número de consumidores de bebida alcoólica nos últimos 30 dias está entre os jovens adultos de 25 a 34 anos, com prevalência do sexo masculino e com maior número de anos de estudo. Nessa tabela também ficou perceptível que, com o passar dos anos (2009-2019), houve uma aderência expressiva do sexo feminino ao álcool e um aumento significante desse consumo na faixa etária que já tem maior porcentagem, a de 25 a 34 anos, subindo de $22,9 \%$ para $26,3 \%$.

No presente estudo percebeu-se uma diminuição substancial da população de fumantes no Brasil, havendo uma queda na porcentagem referente à ambos os sexos e de todas as faixas etárias (a partir da maior idade). Esses dados podem ser corroborados por Portes et al, que em pesquisas no ano de 2018, mostrou que só entre 2009 e 2013 houve uma diminuição de 17,2\% para 14,7\% de tabagistas no país. No entanto, ao se analisar o Atlas de Mortalidade de Câncer, pertencente ao INCA, no período de 2009 a 2018 é visto um aumento da taxa de mortalidade por câncer bucal. Esse aumento pode ser explicado pelo 
fato de o tabaco ser apenas um dos fatores contribuintes para essa neoplasia, como também, a existência de um período de latência das substâncias cancerígenas presentes no cigarro, levando assim muitos anos para se conseguir alcançar um resultado satisfatório de diminuição das mortes.

Entretanto, ao voltar o olhar para a tabela de tabagistas, aqui apresentada, é notório o decréscimo na taxa de pessoas usuárias. E para essa diminuição existem diversas explicações, a principal delas é a criação no final da década de 80 do Programa Nacional de Controle do Tabagismo, um trabalho conjunto do INCA e do Ministério da Saúde, que teve como objetivo reduzir o número de tabagistas no Brasil por meio de ações de educação e prevenção sobre o tema. Em 2005, esse programa passou a ser integrado à Política Nacional de Controle do Tabaco, criado em um Tratado da OMS (Organização Mundial de Saúde) visando combater a epidemia mundial do tabaco. Uma dessas medidas impostas por lei no país, por esse documento, foi a proibição do fumo em locais coletivos e fechados, através do art.49 da Lei 12.546. Outro ato decretado por essa mesma lei, foi o aumento de imposto sobre os cigarros, gerando assim uma enorme carga tributária sobre esse produto e consequentemente seu aumento de preço, além de ter se estabelecido um valor mínimo para sua venda. Algumas outras imposições adotadas como, a proibição da veiculação de publicidade dos cigarros e o uso obrigatório na embalagem em tarja preta "este produto causa câncer" tem contribuído de forma massiva para esse sucesso do Brasil no seu programa de combate ao tabagismo. Aliado a isso, o SUS tem oferecido uma rede de apoio a esses dependentes da nicotina, chegando a atender em média 150 mil pessoas ao ano (Cavalcante et al, 2017).

Em contrapartida, houve um aumento significativo dos consumidores de bebida alcoólica, principalmente entre os mais jovens, e esse dado pode ser fundamentado pelo estudo de Gomes et al em 2019, que mostrou em uma pesquisa com jovens universitários da capital de Fortaleza-CE, que em 5 anos de graduação ocorreu um acréscimo de 3\% no consumo de álcool entre os homens, e de quase 10\% entre as mulheres. Essa crescente é elucidada pela atual situação que o jovem vive ao se encontrar sob pressão já que nessa fase suas responsabilidades aumentam, pois é quando seu futuro desempenho profissional começa a ser cobrado.

Já em relação ao álcool, o Brasil tem investido nos últimos anos em políticas públicas voltadas ao consumo consciente, como exemplo, a Lei 11.705 sancionada em 2008 e popularmente conhecida como "lei seca", que tem como objetivo evitar o consumo alcoólico por quem está dirigindo algum tipo de veículo. Além disso, outras medidas como a Lei 8.069/90 que proíbe a venda de bebidas alcoólicas pelos estabelecimentos para menores de 18 anos, e a restrição de propaganda de bebidas com teor superior a 13 graus Gay Lussac, tem sido reforços nesses programas de conscientização da população sobre o álcool (Brasil, 2015). Porém, as taxas de crescimento de consumidores vão na contramão desses projetos apresentados, e isso pode estar ligado a costumes ainda enraizados na sociedade de normalizar o consumo precoce de bebidas alcoólicas, onde esses adolescentes são levados a essa inicialização ou por curiosidade, ou como uma forma de rebeldia para chamar atenção da família, ou ainda como forma de refúgio dos problemas familiares. E o álcool apesar de ser uma droga lícita é uma droga psicotrópica e uma grande porta de entrada para o consumo do tabaco, por isso a importância do alinhamento dos mesmos nos programas públicos de promoção de saúde (Mangueira et al, 2014).

É importante ressaltar que esses dados apresentados nas tabelas e que tem origem no VIGITEL, podem não ser fiéis a real realidade brasileira, uma vez que, há cinco anos, o último levantamento da PNAD (Pesquisa Nacional por Amostra de Domić́lios) realizado pelo IBGE, indicava que cerca de $91,1 \%$ dos lares possuíam um aparelho celular, e desse número, apenas 37,1\% contava com a telefonia fixa convencional. Atualmente, em pleno 2020, a porcentagem é ainda menor com o número de acessos do serviço decrescendo há mais de 12 meses. A última PNAD Contínua, divulgada em abril, mostrou que somente 28,4\% dos domicílios brasileiros utilizam um telefone fixo (Brasil, 2020). Com esta constante queda, vê-se a necessidade em um futuro breve, de uma reformulação e adaptação do VIGITEL, para continuar a pesquisa não só por meio de linhas fixas, mas também por linhas móveis. 
O presente trabalho também apresentou como limitações a utilização de dados secundários, os quais não proporcionam ao pesquisador o controle dos seus possíveis vieses de informação e confundimento. Além disto, trata-se de um estudo descritivo transversal, onde a causalidade não pode ser fechada, apenas fornece a geração de hipóteses. Apesar disso, os dados são de base nacional e de um importante sistema de informação, o que evidencia a representatividade dos achados.

Com a inclusão, no início dos anos 2000, do cirurgião-dentista na Estratégia Saúde da Família (ESF), ampliou-se o acesso da população, por meio do SUS, as ações assistenciais e de promoção de saúde bucal. Diante disso, Casotti et al afirmou em 2014 que, aliado a essa abertura, o modo como a odontologia vem trabalhando nos últimos anos, focando na prevenção e promoção de saúde, tem contribuído para haver um maior diagnóstico precoce de doenças relacionadas com o meio bucal, principalmente o câncer bucal. E como resultado disso, o sistema tem investido em atividades que possam reduzir ou evitar o surgimento dessas doenças.

Para tornar possível esse diagnóstico de forma precoce, é preciso um conhecimento amplo sobre o assunto. No seu estudo, Souza et al constataram, em 2016, entre os cirurgiões-dentistas avaliados que trabalhavam em unidades básicas de saúde, que a maioria tinha a percepção de si mesmo como profissionais capacitados para o diagnóstico de um possível câncer de boca, a partir da identificação de sinais e sintomas em diferentes estágios de evolução da doença, e para sensibilizar os indivíduos quanto aos fatores de risco.

Um estudo demonstrou que, na conduta clínica em relação ao câncer bucal, quase $80 \%$ dos cirurgiões-dentistas participantes da amostra realizavam o exame clínico investigando a presença de lesões e condições características, na primeira consulta. Com esta proporção de profissionais com um olhar mais minucioso, era de se esperar um maior número de casos da doença diagnosticados precocemente. Porém, o paciente fumante e/ou consumidor severo de bebidas alcoólicas, portador de câncer bucal, possui baixa frequência à consultas odontológicas periódicas, e uma demora na sintomatologia da doença, além de dificuldade de acesso ao serviço de saúde especializado, levando a um diagnóstico tardio da patologia em questão (Falcão, 2010).

Evidências mostraram que, quase $85 \%$ dos dentistas encaminham os casos suspeitos para especialistas ou para uma faculdade de odontologia. É o que diz uma pesquisa sobre o conhecimento dos cirurgiões-dentistas do município de Feira de Santana (BA) - 2006. Onde nem sempre este encaminhamento garante uma rápida realização do procedimento, e em se tratando dessa doença, a demora no diagnóstico reflete num prognóstico desfavorável. Sendo a biópsia um recurso diagnóstico de realização razoavelmente simples, sua execução imediata poderia acelerar o reconhecimento precoce, e consequentemente, melhorar o prognóstico. Contudo, nem todos os cirurgiões-dentistas estão preparados para fazer esse procedimento. Nessa pesquisa ficou perceptível também que, os profissionais da área não possuem o hábito de orientar os seus pacientes em relação ao câncer bucal e autoexame, colaborando assim de certa forma para o diagnóstico tardio, já que se sabe que a realização periódica do autoexame pode ser uma estratégia para o indivíduo perceber precocemente alterações de tecidos e buscar auxílio (Falcão, 2010).

Em um relato de experiência, Bezerra et al no ano de 2016, mostraram que, de fato, ao encontrar lesões suspeitas, os profissionais da atenção básica encaminhávamos pacientes para centros especializados em patologias. No entanto, nos seus resultados, foi evidenciado um combate além-consultório ao câncer bucal, no qual cirurgiões-dentistas e suas equipes participavam de visitas domiciliares, com foco em consumidores de tabaco e álcool e realizavam exames intra e extrabucais para investigação de possíveis casos neoplásicos. Com esse tipo de ação, o número de detecção precoce aumentou, indicando a possibilidade de uma maior taxa de sucesso na cura da doença. Além disso, percebeu-se que essa prática extramuros foi importante não só para a população como também para os próprios profissionais que relataram uma maior sensibilização por parte deles em relação a essa patologia. 
Em uma cartilha lançada pelo Ministério da Saúde em 2008, foi ressaltado que para um diagnóstico precoce é importância não apenas uma boa anamnese, mas também o combate aos fatores de risco causadores do câncer bucal, mostrando assim a necessidade da integração da equipe de saúde bucal da atenção básica aos programas de prevenção e proteção. Uma das atividades primordiais que precisa ser executada por essas equipes de saúde bucal na atenção básica são as ações educativas para detecção de lesões de mucosa e câncer bucal, voltadas para todas as faixas etária, além da realização de exames de forma periódica em pessoas do sexo masculino, acima de 40 anos e que são tabagistas/etilistas ou que estejam expostos com frequência a radiação solar, ou ainda que sejam portadores de alguma deficiência imunológica. A Equipe de Saúde Bucal é chamada também a integrar os programas com as ações citadas acima, além de ficar responsável por informar sistematicamente à população os devidos lugares para a realização do exame de diagnóstico do câncer bucal (Brasil, 2008).

Objetivando a conscientização precoce, o Conselho Federal de Odontologia (CFO) estabeleceu a primeira semana de novembro, segundo a Lei 13.230/2015, como a Semana Nacional de Prevenção do Câncer Bucal, que além de incentivar a população a visitar os cirurgiões-dentistas, busca também conscientizá-la e informá-la sobre a doença, uma vez que, foi constatado que a desinformação por parte dos leigos tem se mostrado um grande desafio.

\section{Conclusão}

O presente estudo demonstrou que a frequência dos tabagistas no Brasil, segundo o VIGITEL, e de acordo com as variáveis de sexo, idade e grau de escolaridade entre os anos de 2009 e 2019 teve um decréscimo expressivo, chegando à 5,7\%. E em sentido contrário a esse sucesso atingido pelo país na diminuição de usuários do tabaco, encontram-se os consumidores de bebida alcoólica, nos últimos 30 dias, que usando das mesmas variáveis e mesma pesquisa, apresentou um aumento significante, principalmente entre os adultos jovens (25-34 anos), chegando a crescer 3,4\% nos últimos 10 anos.

Diante desse trabalho, sugere-se que mais estudos sejam realizados, visto que foi utilizado dados secundários provenientes de pesquisas pré-existentes. Com isso, nota-se a necessidade de uma política pública direcionada para ações que visem a mortalidade pelo câncer bucal, de forma mais precisa quanto ao tratamento o mais precoce possível.

Já na discussão a respeito do papel do cirurgião-dentista na atenção primária, foi possível perceber que existe ainda a necessidade de investimento em políticas públicas que deem sustentação às ações que visem a redução da mortalidade pelo câncer bucal, com enfoque principalmente no tabaco e em especial no álcool, que ao longo desses 10 anos não teve a diminuição esperada de consumidores, como também uma reformulação das práticas do cirurgião-dentista, para que este busque uma constante atualização sobre o nível de conhecimento referente a doença abordada e possa ainda ser precursor do rastreamento da mesma.

\section{Referências}

ACS. (2020). What Are Oral Cavity and Oropharyngeal Cancers? American Cancer Society (ACS). https://www.cancer.org/cancer/oral-cavity-andoropharyngeal-cancer/about/what-is-oral-cavity-cancer.html.

Andrade, J. O. M., Santos, C. A. S. T., \& Oliveira, M. C. (2015). Fatores associados ao câncer de boca: um estudo de caso-controle em uma população do Nordeste do Brasil. Revista Brasileira de epidemiologia, 18; 894-905.

Bezerra, T. A, Almeida, A. V. S., \& Costa, K. N. F. M. (2016). Relato de experiência: estratégia de prevenção do câncer de boca. Revista de APS, 19; (4), 661664.

Brasil. (2020). Instituto Brasileiro de Geografia e Estatística (IBGE). Registro de Câncer de Base Populacional - RCBP. 2020. https://ces.ibge.gov.br/base-dedados/metadados/ministerio-da-saude/registro-de-cancer-de-base-populacional-rcbp.html.

Brasil. (2015). Lei $\mathrm{n}^{\circ}$ 13.106, de 17 de março de 2015. Institui a política nacional de atenção à saúde auditiva. Jusbrasil. https://www.jusbrasil.com.br/topicos/1422485/venda-de-bebida-alcoolica/legislacao.

Brasil. (2015). Lei $\mathrm{n}^{\circ}$ 13.230, de 28 de dezembro de 2015. Institui a semana nacional de prevenção do câncer bucal. Ministérios da Saúde. http://www.planalto.gov.br/ccivil_03/_ato2015-2018/2015/lei/L13230.htm. 
Research, Society and Development, v. 10, n. 8, e39110817278, 2021

(CC BY 4.0) | ISSN 2525-3409 | DOI: http://dx.doi.org/10.33448/rsd-v10i8.17278

Brasil. (2008). Secretaria de Atenção à Saúde. Departamento de Atenção Básica. Saúde Bucal/Ministério da Saúde, Secretaria de Atenção à Saúde, Departamento de Atenção Básica. - Brasília: Ministério da Saúde. Ministério da Saúde. 92 p.- (Série A. Normas e Manuais Técnicos) (Cadernos de Atenção Básica; 17).

Casotti, E. et al. 2014. Atenção em Saúde Bucal no Brasil: uma análise a partir da Avaliação Externa do PMAQ-AB. Saúde em Debate, 38, $140-157$.

Cavalcante, T.M., et al. 2017. Brasil: balanço da Política Nacional de Controle do Tabaco na última década e dilemas. Cadernos de Saúde Pública, 33; (3), 6175

Cedraz, J. S. B., et al. 2016. Estudo clínico-epidemiológico de pacientes com câncer bucal em um período de treze anos. Revista Cubana de Estomatologia, $53 ;(3), 86-96$.

Conselho Federal de Odontologia. (2020). Semana Nacional de Prevenção do Câncer Bucal: diagnóstico precoce pode salvar vidas. https://website.cfo.org.br/semana-nacional-de-prevencao-do-cancer-bucal-diagnostico-precoce-pode-salvar-vidas/.

Falcão, M. M. L., et al. 2010. Conhecimento dos cirurgiões-dentistas em relação ao câncer bucal. RGO. Revista Gaúcha de Odontologia (Online), 58; (1), 2733.

Gomes, I. P. et al. 2019. Fatores Associados à Manutenção do Vício de Fumar e do Consumo de Álcool entre Acadêmicos de Medicina em uma Capital do Nordeste do Brasil. Revista Brasileira de Educação Médica, 43; (1), 55-64.

Hassona, Y., et al. 2015. Mouth cancer awareness and beliefs among dental patients. International dental journal, 65; (1), 15-21.

Instituto Nacional de Câncer (2020). Câncer de boca. https://www.inca.gov.br/tipos-de-cancer/cancer-de-boca.

Mangueira, S. O., et al. 2015. Promoção da saúde e políticas públicas do álcool no Brasil: revisão integrativa da literatura. Psicologia \& sociedade, 27; (1), 157-168.

Martins, A. M. E. B. L., et al. 2015. Maior acesso à informação sobre como prevenir o câncer bucal entre idosos assistidos na atenção primária à saúde. Ciência \& Saúde Coletiva, 20; (7), 2239-2253.

Ogden, G. R. 2018. Alcohol and mouth cancer. British dental journal, 225; (9), 880-883.

Portes, L. H., Machado, C. V., \& Turci, S. R. B. 2018. Trajetória da política de controle do tabaco no Brasil de 1986 a 2016. Cadernos de Saúde Pública, 34; (2), 1-20.

Scully, C., \& Kirby, J. 2014. Statement on mouth cancer diagnosis and prevention. British dental journal, 216; (1), 37-38.

Sociedade Brasileira de Cirurgia de Cabeça e Pescoço SBCCP. (2020) Álcool e cigarro multiplicam risco de câncer de cabeça e pescoço. http://sbccp.org.br/alcool-e-cigarro-multiplicam-risco-do-cancer-de-cabeca-e-pescoco/.

Souza, J. G. S., \& Sá, M. A. B. 2016. POPOFF, Daniela Araújo Veloso. Comportamentos e conhecimentos de cirurgiões-dentistas da atenção primária à saúde quanto ao câncer bucal. Cadernos Saúde Coletiva, 24; (2), 170-177. 